\title{
Application of color temperature controllability in LED lighting environment of highway tunnel
}

\author{
Zhiqiang Liu ${ }^{1}$, Peifeng Li $^{2}$, Haifeng Jiang ${ }^{1}$, Dan Wang ${ }^{1}$ \\ ${ }^{1}$ Research institute of highway ministry of transport, Beijing, China \\ ${ }^{2}$ Yunnan Molin Expressway Construction Headquarters, Kunming, China
}

\begin{abstract}
In recent years, tunnel LED lighting has been widely used in tunnel lighting engineering because of its advantages such as fast start, low energy consumption and good light color controllability. However, at present, the tunnel LED lighting mostly adopts the single dimming control function of light brightness, which does not give full play to the technical advantages of the tunnel LED lighting with two indicators of light brightness and light color temperature control, and does not consider the influence of the color temperature and spectral distribution of the LED light source on the safety and comfort of the tunnel LED lighting environment. Therefore, this paper studies the relationship between the color temperature of tunnel LED lighting source and the visual comfort and visual recognition distance, analyzes the actual impact of the color temperature of LED light source on the tunnel lighting light environment, and puts forward the color temperature index applicable to the entrance and exit sections of tunnel LED lighting, which provides a theoretical basis for building a safe and comfortable "two types" tunnel LED lighting light environment.
\end{abstract}

\section{Introduction}

Lighting is an important measure to ensure the safety of tunnel road operation and improve the traffic efficiency. For a long time, the most widely used lighting source in the field of highway tunnel is high-pressure sodium lamp. Because of the singleness of its emission spectrum, the influence of the color temperature, color rendering index of the light source and the matching relationship with the spectral efficiency function of human eye on the lighting quality is ignored. In the design, evaluation and operation stage, the brightness index becomes the only factor that takes into account the traffic safety and operation cost ${ }^{[1]}$. In recent years, with a large number of new lighting sources such as white LED and electrodeless fluorescent lamp applied in the field of highway lighting tunnel, the difference of color temperature and emission spectrum of the light source has an increasingly significant impact on the driver's visual perception ${ }^{[2]}$. In some lighting projects with new lighting sources, color temperature, color rendering index and other indicators have been included in the design and evaluation scheme. However, as far as the indicator data are concerned, the impact on the driver's visual effect has not yet been established, and the impact of spectral differences of light sources on the perception of human visual brightness under the intermediate visual conditions has been ignored. With the development of the research on the middle vision of human eyes $[3,4]$, more and more attention has been paid to the difference of spectral wavelength response of human eyes in the design and evaluation of highway lighting.

The color temperature of tunnel LED lighting fixture refers to the color change of human eyes under different energy. In the calculation of color temperature, Kelvin is taken as the unit and $0{ }^{\circ}$ Kelvin $=-273^{\circ} \mathrm{C}$ of blackbody radiation as the starting point. Heating the blackbody, with the increase of energy, will enter the field of visible light. For example, at $2800^{\circ} \mathrm{K}$, the color light emitted is the same as the LED bulb, so we say that the color temperature of the LED bulb is $2800^{\circ} \mathrm{K}$. In the field of visible light, the change of color temperature from low color temperature to high color temperature is orange red $>$ White $>$ Blue(Figure 1)

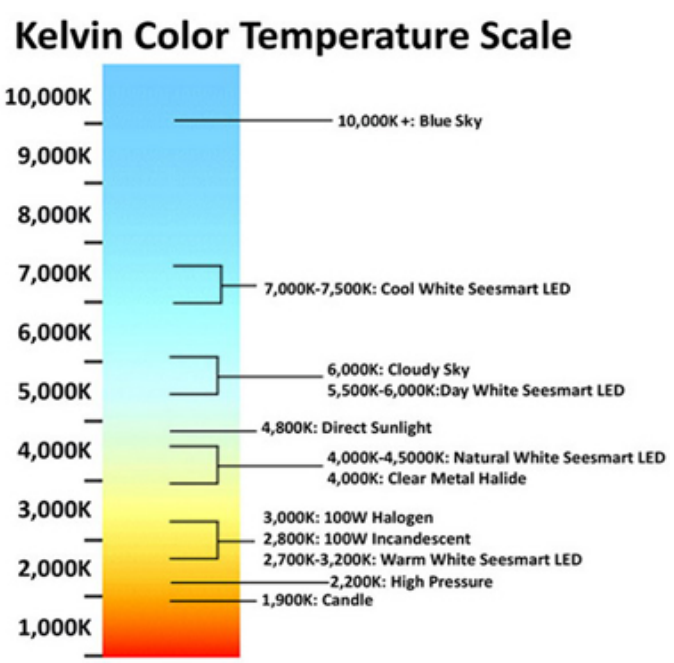

Figure 1 Comparison between color temperature and light color of tunnel LED lamps 
The color temperature of natural light environment changes with the time of day. As shown in Figure 2, the color temperature of the sunlight at the beginning of sunrise and before sunset is about $2000 \mathrm{~K}$. At noon, the sunlight in the sky is very white, and the color temperature is about $5200 \mathrm{k}$. The color temperature of the window light toward the north is about $6500 \mathrm{k}$. The color temperature of the sky in cloudy days is about $7000 \mathrm{~K}$, and the color temperature of the blue sky in sunny days is $12000 \mathrm{k}$.
In artificial light source, the color temperature of ordinary incandescent lamp is about $280 \mathrm{k}$, that of tungsten halogen lamp is $3400 \mathrm{k}$, that of fluorescent lamp is about $6500 \mathrm{k}$, that of warm white fluorescent lamp is about $4500 \mathrm{k}$, and that of high pressure sodium lamp is about $2000 \mathrm{~K} \sim 2100 \mathrm{~K}$. The color temperature of common light sources and their corresponding colors are shown in Table 1.

Table 1 Color temperature of common light source and its corresponding luminous color

\begin{tabular}{|c|c|c|c|c|c|c|c|c|c|}
\hline photochrom & & Red - & 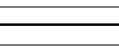 & 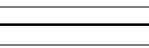 & $\rightarrow$ White - & 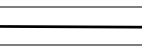 & 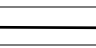 & Cyan & \\
\hline $\begin{array}{c}\text { Color } \\
\text { temperature } \\
(\mathrm{K})\end{array}$ & 1000 & 2000 & 3000 & 4000 & 5000 & 6000 & 7000 & 8000 & 9000 \\
\hline Natural light & & $\begin{array}{l}20 \text { minutes } \\
\text { after sunrise }\end{array}$ & $\begin{array}{c}1 \text { hour } \\
\text { after } \\
\text { sunrise }\end{array}$ & & $\begin{array}{l}\text { Midday } \\
\text { sunshine }\end{array}$ & & $\begin{array}{c}\text { overcas } \\
\text { t sky }\end{array}$ & & \\
\hline Artificial light & candlelight & $\begin{array}{c}\text { Incandescent } \\
\text { lamp }\end{array}$ & $\begin{array}{c}\text { Tungsten } \\
\text { halogen } \\
\text { lamp }\end{array}$ & $\begin{array}{c}\text { White } \\
\text { fluorescent } \\
\text { lamp and } \\
\text { metal } \\
\text { halide lamp }\end{array}$ & $\begin{array}{c}\text { Daylight } \\
\text { fluorescent } \\
\text { lamp }\end{array}$ & $\begin{array}{c}\text { Daylight } \\
\text { fluorescent } \\
\text { lamp }\end{array}$ & & & \\
\hline
\end{tabular}

\section{Study on the relationship between color temperature and visual recognition of tunnel LED lamps}

The lower the color temperature of the light source, the more warm red light, and the higher the color temperature, the more blue light will be. Of course, such light and color will have a certain impact on our psychology and physiology. Therefore, what kind of color light to choose in lighting design is one of the important aspects that designers need to consider. Shilu, Department of natural science, University of Chiba, Japan, through the study of the influence of color temperature of lighting source on the physiological function of human central nervous system [6], the research results are as follows:

(1) High color temperature light source can improve the brain's excitement, concentration, alertness and awakening level, improve the brain activity ability, which is beneficial to the brain load, but high color temperature is unfavorable to the fatigue recovery after the brain load. Long time exposure of high color temperature light source at night can affect people's sleep quality at night, and shorten the four periods of sleep slow wave.

(2) It is recommended that the lighting source with $4000 \mathrm{~K}$ color temperature in the living room is the most comfortable for people, which can make people feel warm, cool and peaceful.

(3) The color temperature and illuminance of light source have mutual effect on people's mood and pleasure. When choosing lighting, we should consider the mutual collocation of these two factors.

Zhang Qingwen, Chen Zhonglin, $\mathrm{Hu}$ Yingkui and Yang Chunyu of Chongqing University in their research on the influence of light source color temperature on the visual effect of tunnel and road lighting show that ${ }^{[7]}$ :
(1) The light source with high color temperature can improve the excitability of human brain, make people concentrate, and thus speed up the response to transient events.

(2) Because low color temperature light source can promote the secretion of melatonin, it is easy to lead to the brain's slow response, and reduce the response speed to transient events.

That is to say, the color temperature of light source has a significant impact on the visual efficacy, which is in line with the concept of "non visual physiological function of human body" proposed by Yasu Kouchi et al. And the research conclusion on "photobiology" abroad.

Since the light with different color temperature can give people different warm and cold feeling, do people like the warm light with low color temperature or the cold light with high color temperature? Research shows that people's preference for light color is mainly related to their activity state (leisure or working state), illumination level and local climate conditions. In addition, it has a certain relationship with people's age and gender. The relationship between light color comfort and illumination level is shown in Figure 3. It can be seen that the tunnel lighting index is generally between $3.0 \mathrm{~cd} / \mathrm{m} 2$ and $180 \mathrm{CD} / \mathrm{m} 2$, and the illumination index is generally between $301 \mathrm{x}$ and $18001 \mathrm{x}$ when considering the asphalt pavement. As shown in the red area in Figure 3, the comfortable color temperature range of people's feeling of light color changes with the change of different illuminance indexes ${ }^{[6]}$.

At present, high pressure sodium lamps with low color temperature are mostly used in highway tunnel and road lighting at home and abroad, which is due to the relatively high luminous efficiency of high pressure sodium lamps under the condition of bright vision. However, the influence of color temperature of light source on visual effect is not considered in the design standards of highway tunnel and road lighting in various countries. Therefore, it is difficult to truly reflect the 
impact of night light environment on human vision, which will cause the evaluation error of lighting quality. In the daytime, the brightness of sunlight will change with time, and the spectrum and color temperature of sunlight will also change with time. Due to the different coverage of clouds, there will also be two different color temperature trends.

\section{Study on safety and comfort performance of light environment}

Based on the above research conclusion, the quality of tunnel LED lighting environment can be achieved. It is a trend to build a safe and comfortable and green lowcarbon tunnel lighting project to realize the intelligent tunnel LED lighting source similar to the change of solar light color temperature through the change of color temperature. The advantages of LED light source can meet the above requirements, such as dynamic color temperature, high color rendering and intelligent brightness adjustment.

The author of this paper, Jiang Haifeng and Yang Yong, used the visual efficacy method to study the effect of different lighting environment conditions on the visual recognition reaction ability of the observer [1], and analyzed the visual recognition performance of the human under different light source color temperature and environmental background brightness conditions. Under the condition of simulated speed of $60 \mathrm{~km} / \mathrm{h}$, the visual recognition reaction time of the observer under five kinds of light sources and different environmental background brightness was tested. The average value of the test results is shown in Table 2 and Figure 6.

Table 2 Visual speed $60 \mathrm{~km} / \mathrm{h}$, the response time of five kinds of light sources with different background brightness

\begin{tabular}{|c|c|c|c|c|c|c|}
\hline \multirow{2}{*}{$\begin{array}{c}\text { Background } \\
\text { luminance } \\
{\left[c d / m^{2}\right]}\end{array}$} & HPS & EF & ${\text { LED }{ }^{\#}}^{\#}$ & LED2 $^{\#}$ & LED3 $^{\#}$ & CV \\
\cline { 2 - 7 } & 3326 & 3242 & 3229 & 3182 & 3122 & 0.0235 \\
\hline 1 & 3303 & 3205 & 3203 & 3162 & 3086 & 0.0246 \\
\hline 2 & 3294 & 3185 & 3190 & 3132 & 3070 & 0.0261 \\
\hline 3 & 3262 & 3166 & 3180 & 3087 & 3054 & 0.0260 \\
\hline 4 & 3232 & 3122 & 3168 & 3054 & 3039 & 0.0257 \\
\hline 5 & & & &
\end{tabular}

The results show that: with the increase of the background brightness, the visual recognition reaction time of the observer decreases continuously; while under the same background brightness, the visual recognition reaction time of the non-polar fluorescent lamp and white LED is less than that of the high pressure sodium lamp, and the reaction time of the high color temperature white LED is less than that of the low color temperature white LED.

\section{Demonstration project test and empirical analysis}

Based on the above research results, through cooperation with relevant technical manufacturers inside and outside the industry, the project team has developed and completed the light and color dual index adjustable tunnel LED lighting fixture, light and color dual index adjustable control system and light and color dual index adjustable drive control power supply, and has carried out test and analysis, verifying the feasibility of the relevant technical scheme. The control algorithm of tunnel LED lighting with adjustable color temperature and brightness is developed.

On the spot, the ambient light color temperature value of the day was also tested. In the morning, noon and noon and afternoon of rainy days, the color temperature changed from $4995 \mathrm{k}$ to $6234 \mathrm{kz}$ (test results at the same tunnel entrance), and the difference was about $1200 \mathrm{k}$. Therefore, the project team combined with the subjective visual evaluation and comfort of the color temperature and light environment outside the tunnel under different natural light environment conditions, decided to adjust the color temperature control according to the ambient light intensity outside the tunnel. The project team then conducted visual recognition test on the small gray square box $(40 \mathrm{~cm} \times 40 \mathrm{~cm})$ arranged in the lane of the tunnel at a stopping sight distance (about $110 \mathrm{~m}$ ) from the tunnel entrance. The visual recognition effect and clarity of the small square box under different color, temperature and light environment were tested in turn. The test results show that the adjustment values of color temperature of each lighting section in the tunnel under different natural light environment outside the tunnel portal are shown in Table 3.

Table 3 Value table of color temperature regulation

\begin{tabular}{|l|l|c|}
\hline $\begin{array}{c}\text { Weather } \\
\text { conditions }\end{array}$ & \multicolumn{1}{|c|}{ Time slot } & \multicolumn{1}{|c|}{$\begin{array}{c}\text { Color temperature } \\
\text { value (k) }\end{array}$} \\
\hline \multirow{3}{*}{ Sunny day } & Day: 11:00-14:00 & 5000 \\
\cline { 2 - 3 } & $\begin{array}{l}\text { Day: } \\
\text { Spring and summer: 6:00-11:00 and 14:00-18:00 }\end{array}$ & 4300 \\
& $\begin{array}{l}\text { Autumn and winter: 7:00-11:00and14:00-17:00 } \\
\text { yy }\end{array}$ & \\
\hline
\end{tabular}




\begin{tabular}{|c|l|c|}
\hline $\begin{array}{c}\text { Rainy and foggy } \\
\text { day }\end{array}$ & $\begin{array}{l}\text { All days } \\
\text { Spring and summer: 6:00-18:00 } \\
\text { Autumn and winter: 7:00-17:00 }\end{array}$ & 3200 \\
\hline \multirow{3}{*}{ Night } & $\begin{array}{l}\text { All nights } \\
\text { Spring and summer: 18:00-6:00 } \\
\end{array}$ & 3500 \\
\hline
\end{tabular}

When the color temperature adjustment control algorithm is applied in the actual project, it can adopt semi-automatic control mode, judge manually for remote control adjustment, or judge the weather conditions according to the brightness test results of the brightness sensor installed outside the tunnel, and the software control algorithm can automatically calculate the color temperature adjustment value.

\section{Conclusion}

Based on the physiology of human visual recognition, this paper systematically studies and analyzes the influence of color temperature on the visual recognition and comfort of tunnel LED lighting fixtures, studies and opens the control system of light color dual index adjustable tunnel LED lighting fixtures, light color dual index adjustable control system and light color dual index adjustable drive control power supply, and successfully carries out a lot of tests, demonstrating the application results of standard coal, color temperature adjustable tunnel The road led lighting system, combined with the light environment inside and outside the tunnel, can effectively alleviate the visual recognition ability of the human eye due to the sudden change of the light lighting environment, improve the visual recognition ability of the human eye to the road conditions at the entrance of the tunnel, and adjust the color, temperature and light environment inside the tunnel in combination with the different light environment conditions outside the tunnel, such as sunny, cloudy, rainy, daytime and night The user provides more comfortable and humanized lighting services, which helps to improve the visibility and comfort level of tunnel LED lighting system.

\section{Reference}

1. Yang Yong, Han Wenyuan, Jiang Haifeng. Performance analysis of highway lighting source based on visual efficacy method. Spectroscopy and spectral analysis. 2015, Vol.35, no.10:2686-2690

2. Jin Peng, Yu Chun Yu, Zhou Qi Feng, et al. Optics and precision engineering, 2011, 19 (1): 51-55

3. Ikeda M S H. Journal of the Optical Society of America., 1981,3(1)71-75.

4. Sagawa K, Takeichi K. Journal of the Optical Society of America A: Optics and Image cience, 1986,3(1):71-75.

5. Liu Yingying, Zhang Qingwen, $\mathrm{Hu}$ Yingkui.LED light source color temperature influence on tunnel lighting entrance section and middle section. Journal of lighting engineering, 2013, Vol. 24 No. 2:30-34
6. Shi Lu. Influence of color temperature of lighting source on physiological function of human central nervous system. Ergonomics, 2006, Vol. 12 No. 2:46-50

7. Zhang Qingwen, Chen Zhonglin, Hu Yingkui, Yang Chunyu. Study on the influence of light source color temperature on visual efficacy of tunnel and road lighting. Journal of lighting engineering. 2008, Vol. 19, No. 2:24-30 